\title{
CARACTERIZAÇÃO DA MORFOLOGIA RADICULAR DE GENÓTIPOS CONTRASTANTES DE SORGO EM BAIXO E ALTO NÍVEIS DE FÓSFORO
}

MICHEL CASTTELANI DA ROCHA ${ }^{1}$, GLAUCO VIEIRA MIRANDA ${ }^{2}$, MARIA JOSÉ VILAÇA VASCONCELOS ${ }^{3 *}$, PAULO CÉSAR MAGALHÃES ${ }^{3}$, GERALDO AFONSO DE CARVALHO JÚNIOR ${ }^{4}$, LIDIANNE ASSIS SILVA 6 , MARCELO OLIVEIRA SOARES ${ }^{5}$, FERNANDO RODRIGO OLIVEIRA CANTÃO ${ }^{6}$, FABRÍCIO RODRIGUES ${ }^{6}$ e ROBERT EUGENE SCHAFFERT ${ }^{3}$

* autor para correspondência

${ }^{1}$ Bolsista FAPEMIG/Embrapa Milho e Sorgo, Mestre em Fitotecnia, Universidade Federal de Viçosa. CEP. 35700-000, Sete Lagoas, MG. E-mail: michelcastelani@yahoo.com.br

${ }^{2}$ Prof ${ }^{\circ}$. Associado, Departamento de Fitotecnia, Universidade Federal de Viçosa, bolsista CNPq-36570-000 Viçosa, MG. E-mail: glaucovmiranda@ufv.br

${ }^{3}$ Pesquisador Embrapa Milho e Sorgo - CEP 35700-000, Sete Lagoas, MG. E-mail: schaffer@ cnpms.embrapa.br,

${ }^{4}$ Graduando em Agronomia, Universidade Federal de Viçosa, Viçosa, $M G$

${ }^{5}$ Doutorando em Genética e Melhoramento, Universidade Federal de Viçosa, Viçosa, MG.

${ }^{6}$ Bolsista Embrapa Milho e Sorgo, CEP. 35700-000, Sete Lagoas, MG.

Revista Brasileira de Milho e Sorgo, v.9, n.1, p. 65-78, 2010

RESUMO - A baixa disponibilidade de fósforo (P) no solo é um dos principais fatores que restringem o desenvolvimento e a produção das plantas. Para a adaptação a esses ambientes, as plantas desenvolveram mecanismos que variam entre espécies e entre genótipos. A capacidade das plantas de explorar o solo, converter formas não utilizáveis de $\mathrm{P}$ em absorvíveis, formar associação com fungos micorrízicos, entre outras características, pode determinar a eficiência das plantas nesses ambientes. Este trabalho teve como objetivo avaliar características morfológicas radiculares relacionadas aos mecanismos de aquisição de fósforo em linhagens de sorgo pertencentes ao programa de melhoramento de sorgo da Embrapa. Foram caracterizadas nove linhagens, quanto à morfologia do sistema radicular, quando cultivadas em solos apresentando dois níveis de fósforo ( 5 e $20 \mathrm{mg} \mathrm{dm}^{-3}$ ). Para essa caracterização, foi utilizado o analisador de imagens WinRhizo v. 4.0. Para as características relatadas como relevantes na aquisição de fósforo, comprimento total de raiz (CTR), comprimento de raiz muito fina (CRMF) e fina (CRF), área de superfície total de raiz (ATR), área de superfície de raiz muito fina (ARMF) e fina (ARF) e número de ramificações (NR), verificou-se variabilidade genética somente quando as plantas foram cultivadas em baixa disponibilidade de fósforo, 
sugerindo que diferentes mecanismos de aquisição podem estar atuando nos ambientes de baixa e alta disponibilidade do nutriente. O comprimento e a área de superfície das raízes apresentaram-se mais importantes na adaptação das plantas em baixa disponibilidade de fósforo que o volume e o diâmetro médio das raízes. As características comprimento específico (CE) e densidade de tecido de raiz (DeTR) podem estar relacionadas com a eficiência das linhagens em baixo fósforo. Essas características podem ter grande utilidade como parâmetros para seleção de genótipos em programas de melhoramento de sorgo.

Palavras-chave: Sorghum bicolor, morfologia de raiz, WinRhizo, eficiência de fósforo, aquisição de fósforo.

\title{
CHARACTERIZATION OF ROOT MORPHOLOGY IN CONTRASTING GENOTYPES OF SORGHUM AT LOW AND HIGH PHOSPHORUS LEVEL
}

\begin{abstract}
Low availability of phosphorus in the soil is one of the principal limiting factors in plant development and plant production. Plants have developed adaptive mechanisms for these environments that vary among species and genotypes within a species. The capacity of plants to explore the soil, transform unusable P forms to usable forms, develop relationships with mycorrhiza fungi, among other traits, influence the plant efficiency in these environments. The objective of this study was to evaluate morphological root characteristics related to mechanisms of phosphorus acquisition in sorghum lines of the sorghum improvement program at Embrapa. Nine lines were used to study the root system in a soil with two levels of phosphorus ( 5 and $20 \mathrm{mg} \mathrm{dm}^{-3}$ ). For this characterization a WinRhizo v. 4.0 image analyzer was used. Genetic variability was observed at low phosphorus levels for total root length, very fine root length, fine root length, total root surface area, root surface area of very fine root, root surface area of fine root and number of root branches, suggesting that different mechanisms of phosphorus acquisition may be occurring in environments with low and high phosphorus availability. Root length and root surface area appear to be more important for plant adaptation to low phosphorus levels than root volume and root diameter. Specific root length and root tissue density were the most useful parameters related to plant productivity at low phosphorus. These characteristics could be useful parameters for selection in sorghum improvement programs.
\end{abstract}

Key Words: Sorghum bicolor, Root morphology, WinRhizo, Phosphorus efficiency, Phosphorus acquisition.

Revista Brasileira de Milho e Sorgo, v.9, n.1, p. 65-78, 2010

Versão impressa ISSN 1676-689X / Versão on line ISSN 1980-6477 - http://www.abms.org.br 
Dos macronutrientes essenciais às plantas, o P é um elemento que está frequentemente em concentrações subótimas para as culturas na solução do solo. Isso é consequência de sua habilidade de formar compostos de alta energia de ligação com os colóides, conferindo-lhe alta estabilidade na fase sólida (Novais \& Mello, 2007). Dessa forma, mesmo que os teores totais do fósforo no solo sejam altos em relação aos necessários para as plantas, apenas uma pequena fração está disponível às plantas (Liu et al., 2006; Parentoni, 2008).

Devido às novas tendências socioeconômicas e ambientais, a busca por culturas mais eficientes na aquisição e uso de $\mathrm{P}$ e outros nutrientes apresenta razões básicas: os fertilizantes são os insumos com a maior participação no custo final da produção agrícola em escala comercial, sendo que seu uso é, na maioria das vezes, restringido pelo pequeno produtor, devido à falta de recursos (Good et al., 2004; Parentoni, 2008). O uso de fertilizantes em altas doses pode causar grande impacto ambiental, como a contaminação dos mananciais hídricos, fato já verificado para $\mathrm{N}$ e $\mathrm{P}$ em áreas de agricultura intensiva (Sharpley et al., 2001; Parentoni, 2008). Os fertilizantes são obtidos a partir de combustíveis fósseis $(\mathrm{N})$ ou dependem de recursos minerais $(\mathrm{P})$ que devem se esgotar em um período relativamente curto (Clarkson \& Hawkesford, 1993; Parentoni, 2008). Até 80 a $90 \%$ do P aplicado como fertilizante pode ser adsorvido pelas partículas do solo, tornando, assim, essa fração não disponível às plantas (Gerke et al., 1994; Lambers et al., 2006).

As plantas, de maneira geral, apresentam vários mecanismos e processos que contribuem para a aquisição e o uso eficiente de $\mathrm{P}$ e outros nutrientes que se encontram pouco disponíveis no solo. Esses mecanismos e processos estão relacionados com a expressão de características morfológicas e fisiológicas desejáveis. Estes mecanismos de adaptação são encontrados diferentemente entre espécies e/ou cultivares, indicando existência de variabilidade genética para aquisição e utilização de nutrientes (Fageria, 1998). Genótipos eficientes na aquisição de nutrientes pouco móveis no solo, como o $\mathrm{P}$, podem ter um aumento na capacidade de exploração do solo, por meio de modificações na morfologia do sistema radicular e podem converter as formas não utilizáveis para formas absorvíveis, por meio da exsudação de compostos orgânicos ou de associação com microorganismos (Rengel \& Marschner, 2005).

O objetivo deste trabalho foi avaliar características morfológicas radiculares relacionadas aos mecanismos de aquisição de fósforo em linhagens de sorgo.

\section{Material e Métodos}

\section{Recursos genéticos}

Foram utilizadas, para o estudo das características morfológicas do sistema radicular, nove linhagens de sorgo que fazem parte do Programa de Melhoramento de Sorgo da 
Embrapa Milho e Sorgo. Dessas nove linhagens, oito foram anteriormente classificadas quanto à eficiência e resposta de produtividade de grãos em dois níveis de disponibilidade de P no solo (Schaffert et al., 2001).

\section{Cultivo de linhagens e coleta de plantas para análise}

As linhagens de sorgo foram avaliadas na Embrapa Milho e Sorgo, em Sete Lagoas, MG, localizada a $19^{\circ} 28^{\prime} \mathrm{S}$ e $44^{\circ} 15^{\prime} 08^{\prime \prime} \mathrm{W}$ na altitude de $732 \mathrm{~m}$, apresentando clima, segundo Köopen, do tipo Aw (clima de savana com inverno seco). O período de cultivo para a fenotipagem das plantas foi de novembro a dezembro de 2007, sendo o experimento instalado em dois canteiros, um em solo com baixa disponibilidade de fósforo $\left(5 \mathrm{mg} \mathrm{dm}^{-3}\right) \mathrm{e}$ outro com alta disponibilidade $\left(20 \mathrm{mg} \mathrm{dm}^{-3}\right)$, envolvidos por um telado de sombrite $50 \%$. Cada canteiro media $12,0 \mathrm{~m}$ de comprimento x 1,2 m de largura x 0,4 m de profundidade.

$O$ plantio das linhagens foi realizado manualmente, em blocos casualizados, com três repetições. Cada parcela experimental foi composta por duas linhas de 1,2 $\mathrm{m}$ de comprimento, com espaçamento de 0,20 m entre plantas e entre linhas. A irrigação, quando necessária, foi realizada por aspersão. Para a adequação dos nutrientes, foi feita uma adubação com sulfato de amônio e cloreto de potássio no plantio e uma de cobertura com ureia, seguindo a recomendação para adubação de sorgo (Rodrigues, 2007).
Foram coletadas três plantas por tratamento, aos 40 dias de cultivo após a emergência (DAE), para a caracterização morfológica. Com auxílio de uma espátula, foi removida a planta inteira, parte aérea e sistema radicular, do solo e transferida para uma peneira 60 Mesh, para evitar a perda das raízes. Em seguida, foi separada a parte aérea do sistema radicular. A parte aérea das três plantas foi levada para estufa de circulação forçada, a $72^{\circ}$ $\mathrm{C}$, até atingir peso constante, para determinação de massa seca (MSPA - g 3plantas ${ }^{-1}$ ). O sistema radicular, ainda aderido a blocos de solo, foi transferido para uma peneira de 20 Mesh e lavado com água corrente. Em seguida, as raízes das três plantas foram armazenadas em etanol $70 \%$ até o dia da avaliação de sua morfologia, quando foram retiradas do álcool e colocadas em uma bandeja contendo água destilada.

\section{Avaliação da morfologia radicular de linhagens de sorgo}

As características do sistema radicular foram avaliadas com o analisador de imagens WinRhizo v. 4.0, Regent Systems, Quebec (Canadá), acoplado a scanner profissional Epson XL 10000 equipado com unidade de luz adicional (TPU), seguindo os procedimentos de análise sugeridos por Bouma et al. (2000).

Foram determinadas as seguintes características: Comprimento total de raiz (CTR) (cm), área de superfície total (ATR) $\left(\mathrm{cm}^{2}\right)$, diâmetro médio de raiz $(\mathrm{DMR})(\mathrm{mm})$, volume total de raiz (VTR) $\left(\mathrm{cm}^{3}\right)$, número de 
ramificações (NR), comprimento de raiz muito fina (CRMF) $(\mathrm{cm})$, comprimento de raiz fina $(\mathrm{CRF})(\mathrm{cm})$, comprimento de raiz grossa $(\mathrm{CRG})$ $(\mathrm{cm})$, área de superfície de raiz muito fina (ARMF) $\left(\mathrm{cm}^{2}\right)$, área de superfície de raiz fina (ARF) $\left(\mathrm{cm}^{2}\right)$, área de superfície de raiz grossa (ARG) $\left(\mathrm{cm}^{2}\right)$, comprimento específico (CE) $(\mathrm{cm}$ $\left.\mathrm{g}^{-1}\right)$, finura (Fineness) de raiz (FR) $\left(\mathrm{cm} \mathrm{cm}^{-3}\right) \mathrm{e}$ densidade de tecido de raiz (DeTR) $\left(\mathrm{g} \mathrm{cm}^{-3}\right)$.

As características de comprimento e área de superfície por classes de diâmetro (d) (muito finas $\mathrm{d}<0,5 \mathrm{~mm}$; finas $0,5 \mathrm{~mm}<\mathrm{d}<2 \mathrm{~mm}$ e grossas $\mathrm{d}>2 \mathrm{~mm}$ ) foram determinadas segundo a classificação proposta por Bhom (1979). Após a avaliação do sistema radicular, as raízes foram levadas para estufa, para determinação de massa seca de raiz (MSR) (g 3plantas ${ }^{-1}$ ), massa seca total (MST) (g 3plantas $\left.{ }^{-1}\right)$ e relação raiz/parte aérea (MSR/MSPA), conforme citado para parte aérea. Os dados coletados, de todas as características, foram submetidos à análise de variância individual por nível de $\mathrm{P}$ e conjunta $(\mathrm{p}<0,05)$ e comparações de médias, pelo teste de Tukey $(\mathrm{p}<0,05)$.

\section{Resultados e Discussão}

A interação genótipo e ambiente não foi significativa para nenhuma das características avaliadas (Tabela 1), indicando que o nível de fósforo tem efeito na expressão das características avaliadas, independentemente do genótipo.

Diferenças significativas entre o baixo e o alto nível de fósforo foram verificadas para as características CTR, ATR, DMR, NR, CRMF, ARMF, MSR, MSR/MSPA, CE, FR e DeTR (Tabela 1), indicando que os genótipos responderam diferentemente, em relação a essas características, à quantidade de fósforo dos solos onde foram cultivadas.

Para as características DMR, CRG, ARG, ARF, MSPA, MSR, MST e FR, os genótipos apresentaram diferenças significativas, independentemente do nível de fósforo (Tabela 1), indicando a existência de variabilidade genética para essas características. Entretanto, quando as plantas se desenvolvem em ambiente desfavorável quanto ao suprimento de nutrientes, as raízes podem reagir a essa adversidade apresentando alta plasticidade fenotípica (Fitter, 1991; Marschner, 1995). Isso pode ser verificado pela ausência de diferença significativa entre as linhagens para DMR, CRG, ARG, MSPA, MSR, MST e FR, quando as plantas foram crescidas no ambiente de baixa disponibilidade de fósforo (Tabela 4), mostrando que as linhagens tiveram um efeito de ambiente modificando a expressão fenotípica dessas características, tornando-as estatisticamente semelhantes entre as linhagens.

As médias das características CTR, ATR, CRMF, ARMF, NR, CE, FR para os genótipos cultivados em baixo nível de fósforo foram menores que as médias do alto fósforo (Tabela 2). Segundo Desnos (2008), o crescimento de raiz primária e raízes laterais é restringido quando elas encontram um meio com baixo nível de Pi. Essa inibição no crescimento é resultante da 


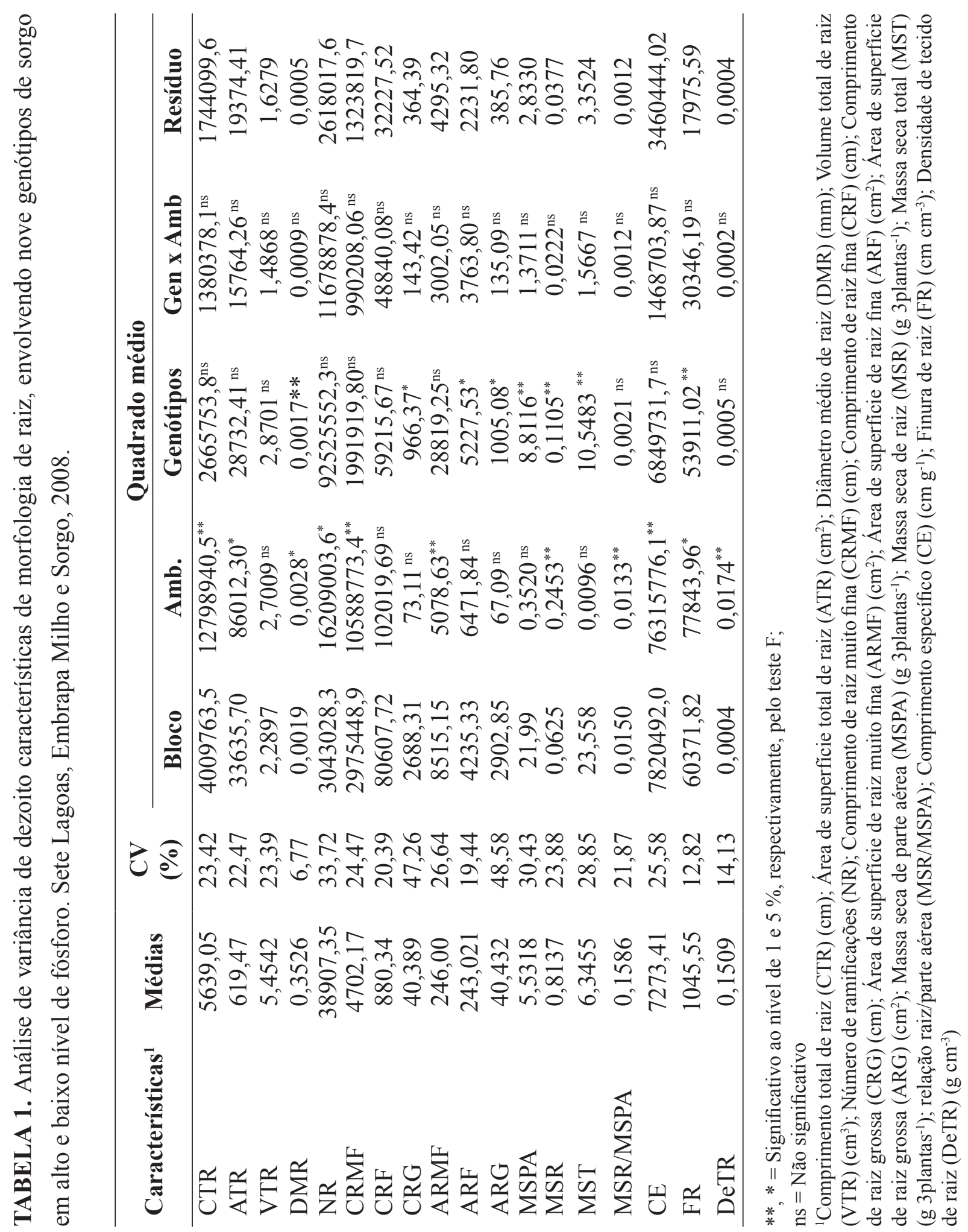


reduzida elongação das células (Williamson et al., 2001; Reymond et al., 2006) e da atividade meristemática (Sanchez-Calderon et al., 2005; Jain et al., 2007b), sendo esse efeito irreversível quando a deficiência de fósforo é mantida por muitos dias (Sanchez-Calderon et al., 2005; Jain et al., 2007b). Assim, como essas características estão relacionadas com o alongamento da raiz, a inibição de seu crescimento reduz os valores de todas estas características em relação ao nível de alta disponibilidade de fósforo.

Para as características DMR, MSR, MSR/ MSPA e DeTR, as médias no baixo fósforo foram maiores que as médias do alto P (Tabela 2). O aumento na partição de carboidratos em direção à raiz e a inibição do crescimento da parte aérea podem resultar em um aumento na MSR e na razão raiz/parte aérea em plantas deficientes em fósforo (Khamis et al., 1990; Marschner, 1995). A DeTR (MSR/VTR) varia de forma direta com a MSR; portanto, o aumento da massa seca de raiz pode ser acompanhado de um aumento na DeTR. O DMR não apresenta uma resposta universal quanto à sua diminuição ou aumento em baixa disponibilidade de fósforo (Schroeder \& Janos, 2005; Lambers et al., 2006). Gahoonia \& Nielsen (2004) relatam que, possivelmente devido à dificuldade de avaliação, há poucos trabalhos que apresentam medidas de DMR.

As linhagens apresentaram diferenças significativas para as características DMR, CRG, ARG, MSPA, MSR, MST, FR (Tabela 3) apenas no canteiro contendo alta disponibilidade de fósforo, indicando que as linhagens apresentaram variabilidade para a realização de seleção de plantas com base nessas características apenas no ambiente de alto fósforo. As raízes grossas ( $>2 \mathrm{~mm}$ diâmetro) estão ligadas ao suporte e ancoragem da planta e absorvem pequenas quantidades de água e nutrientes (Wilcox et al., 2004). O DMR é uma característica relacionada à aquisição de nutrientes (Gahoonia \& Nielsen, 2004). Entretanto, é menos importante na absorção de fósforo que o comprimento de raiz (Casper \& Jackson, 1997; Schroeder\& Janos, 2005). O fato de as linhagens de sorgo testadas não apresentarem diferenças significativas no ambiente de alto fósforo, para as características da morfologia radicular (CTR, CRMF, CRF, ATR, ARMF, ARF), que são relatadas como relevantes na aquisição de fósforo, indica a possibilidade de que outros mecanismos de aquisição estejam relacionados com a resposta das linhagens ao ambiente de alto fósforo. Os resultados encontrados neste trabalho são compatíveis com os encontrados para milho por Parentoni (2008), o qual sugere mecanismos diferentes de aquisição de fósforo atuando nos ambientes de alto e baixo fósforo.

Pelos resultados apresentados na Tabela 4, verifica-se que existem diferenças significativas para as características CTR, ATR, NR, CRMF, CRF, ARMF e ARF entre as linhagens, quando cultivadas em baixo fósforo (Tabela 4). Esses resultados indicam a existência de variabilidade em sorgo para essas características do sistema radicular, quando cultivadas em ambientes 
TABELA 2. Médias das características CTR, ATR, CRMF, ARMF, DMR, NR, MSR, MSR/MSPA, CE, FR e DeTR. Análise das médias entre os ambientes de alta e baixa disponibilidade de fósforo, pelo Teste de $\mathrm{F}(\mathrm{p}<0,05)$.

\begin{tabular}{lcc}
\hline \multirow{2}{*}{ Características } & \multicolumn{2}{c}{ Médias $^{1}$} \\
\cline { 2 - 3 } CTR & Baixo P (Ambiente 1) & Alto P (Ambiente 2) \\
ATR & $5152,21 \mathrm{~B}$ & $6125,90 \mathrm{~A}$ \\
CRMF & $579,56 \mathrm{~B}$ & $659,38 \mathrm{~A}$ \\
ARMF & $4259,36 \mathrm{~B}$ & $5144,99 \mathrm{~A}$ \\
DMR & $222,91 \mathrm{~B}$ & $269,11 \mathrm{~A}$ \\
NR & $0,360 \mathrm{~A}$ & $0,345 \mathrm{~B}$ \\
MSR & $34767,99 \mathrm{~B}$ & $43046,72 \mathrm{~A}$ \\
MSR/MSPA & $0,881 \mathrm{~A}$ & $0,746 \mathrm{~B}$ \\
CE & $0,174 \mathrm{~A}$ & $0,143 \mathrm{~B}$ \\
FR & $6084,61 \mathrm{~B}$ & $8462,22 \mathrm{~A}$ \\
DeTR & $1007,58 \mathrm{~B}$ & $1083,52 \mathrm{~A}$ \\
\hline
\end{tabular}

${ }^{1}$ Médias seguidas de mesma letra na horizontal não diferem significativamente pelo teste $\mathrm{F}(\mathrm{p}<0,05)$. Comprimento total de raiz (CTR) (cm); Área de superfície total de raiz (ATR) $\left(\mathrm{cm}^{2}\right)$; Diâmetro médio de raiz (DMR) (mm); Número de ramificações (NR); Comprimento de raiz muito fina (CRMF) (cm); Área de superfície de raiz muito fina (ARMF) $\left(\mathrm{cm}^{2}\right)$; Massa seca de raiz (MSR) (g 3plantas $\left.{ }^{-1}\right)$; Massa seca raiz/parte aérea (MSR/MSPA); Comprimento específico (CE) $\left(\mathrm{cm} \mathrm{g}^{-1}\right)$; Finura de raiz (FR) $\left(\mathrm{cm} \mathrm{cm}^{-3}\right)$; Densidade de tecido de raiz (DeTR) $\left(\mathrm{g} \mathrm{cm}^{-3}\right)$

com baixo nível de fósforo, e que a expressão dessas características resulta na plasticidade morfológica que as raízes apresentam para aumentar a captação de nutrientes. Assim, essas características podem ser mais bem exploradas em programas de melhoramento genético que buscam melhor entender os mecanismos de adaptação de sorgo sobre estresse de fósforo. A seleção de plantas com base nessas características deve ser realizada em ambientes com baixa disponibilidade de fósforo. Esses resultados (Tabela 4) sugerem também que o comprimento e a área de superfície das raízes apresentaram-se mais importantes na adaptação das plantas em baixa disponibilidade de fósforo que o volume e o diâmetro médio das raízes, que não foram significativamente diferentes entre as linhagens estudadas.

As médias de CTR, ATR, CRMF, CRF, ARMF, ARF e NR das nove linhagens cultivadas em baixo fósforo estão apresentadas na Tabela 5. Observa-se que a linhagem 
TABELA 3. Análise de variância de dezoito características de morfologia de raiz envolvendo nove genótipos de sorgo, em alto nível de fósforo. Sete Lagoas, Embrapa Milho e Sorgo, 2008.

\begin{tabular}{lcrrr}
\hline \multirow{2}{*}{ Características $^{1}$} & Médias & CV (\%) & \multicolumn{2}{c}{ Quadrado médio } \\
\cline { 4 - 5 } & & & Genótipos & \multicolumn{1}{c}{ Resíduo } \\
\hline CTR & 6125,90 & 26,65 & $2017407,42^{\text {ns }}$ & 2665177,04 \\
ATR & 659,3825 & 24,66 & $21432,85^{\text {ns }}$ & 26430,09 \\
VTR & 5,6778 & 23,75 & $2,1515^{\text {ns }}$ & 1,8181 \\
DMR & 0,3454 & 5,41 & $0,0013^{* *}$ & 0,0003 \\
NR & 43046,72 & 33,72 & $177601550,2^{\text {ns }}$ & 210663309,11 \\
CRMF & 5144,99 & 27,87 & $1492727,88^{\text {ns }}$ & 2056690,29 \\
CRF & 923,81 & 22,13 & $57549,75^{\text {ns }}$ & 41797,77 \\
CRG & 39,2261 & 31,32 & $476,2672^{*}$ & 150,9716 \\
ARMF & 269,1094 & 30,59 & $3249,05^{\text {ns }}$ & 6774,67 \\
ARF & 253,9693 & 20,31 & $5004,26^{\text {ns }}$ & 2661,86 \\
ARG & 39,3180 & 27,55 & $451,6208^{* *}$ & 117,3202 \\
MSPA & 5,6125 & 20,57 & $6,4495^{* *}$ & 1,3326 \\
MSR & 0,7462 & 21,33 & $0.0625^{*}$ & 0,0253 \\
MST & 6,3588 & 19,50 & $7,5629^{* *}$ & 1,5371 \\
MSR/MSPA & 0,1429 & 22,40 & $0,0013^{\text {ns }}$ & 0,0010 \\
CE & 8462,21 & 27,77 & $5933686,24^{\text {ns }}$ & 5520724,57 \\
FR & 1083,52 & 9,92 & $48543,14^{* *}$ & 11560,79 \\
DeTR & 0,1329 & 17,50 & $0,00029^{\text {ns }}$ & 0,00055 \\
\hline
\end{tabular}

**,* = Significativo ao nível de 1 e $5 \%$, respectivamente, pelo teste $\mathrm{F}$; ns = Não significativo

${ }^{1}$ Comprimento total de raiz $(\mathrm{CTR})(\mathrm{cm})$; Área de superfície total de raiz (ATR) $\left(\mathrm{cm}^{2}\right)$; Diâmetro médio de raiz (DMR) (mm); Volume total de raiz (VTR) $\left(\mathrm{cm}^{3}\right)$; Número de ramificações (NR); Comprimento de raiz muito fina $(\mathrm{CRMF})(\mathrm{cm})$; Comprimento de raiz fina $(\mathrm{CRF})(\mathrm{cm})$; Comprimento de raiz grossa $(\mathrm{CRG})(\mathrm{cm})$; Área de superfície de raiz muito fina (ARMF) $\left(\mathrm{cm}^{2}\right)$; Área de superfície de raiz fina (ARF) $\left(\mathrm{cm}^{2}\right)$; Área de superfície de raiz grossa $(\mathrm{ARG})\left(\mathrm{cm}^{2}\right)$; Massa seca de parte aérea (MSPA) (g 3plantas $\left.{ }^{-1}\right)$; Massa seca de raiz (MSR) (g 3plantas $\left.{ }^{-1}\right)$; Massa seca total (MST) (g 3plantas ${ }^{-1}$ ); relação raiz/parte aérea (MSR/MSPA); Comprimento específico (CE) $\left(\mathrm{cm} \mathrm{g}^{-1}\right)$; Finura de raiz (FR) $\left(\mathrm{cm} \mathrm{cm}^{-3}\right)$; Densidade de tecido de raiz (DeTR) $\left(\mathrm{g} \mathrm{cm}^{-3}\right)$.

BR005R, mais produtiva, apresentou as maiores médias para essas características e a linhagem ATF06B, menos produtiva, apresentou as menores médias. As linhagens eficientes SC283, BR008B e ATF40B e as ineficientes BR007B e BR001B apresentaram valores de médias próximos e estatisticamente semelhantes. Entretanto, as linhagens BR008B e ATF40B apresentaram uma tendência a ter menores valores de massa seca de raiz (Tabela 
TABELA 4. Análise de variância de dezoito características de morfologia de raiz envolvendo nove genótipos de sorgo, em baixo nível fósforo. Sete Lagoas, Embrapa Milho e Sorgo, 2008.

\begin{tabular}{lcccc}
\hline \multirow{2}{*}{ Características $^{1}$} & \multirow{2}{*}{ Médias } & \multirow{2}{*}{ CV (\%) } & \multicolumn{2}{c}{ Quadrado médio } \\
\cline { 4 - 5 } CTR & & Genótipos & Resíduo \\
ATR & 5152,21 & 16,87 & $2028724,48^{*}$ & 755585,83 \\
VTR & 579,56 & 16,27 & $23063,83^{*}$ & 8898,15 \\
DMR & 5,2305 & 18,43 & $2,2054^{\text {ns }}$ & 0,9296 \\
NR & 0,3598 & 7,22 & $0,0013^{\text {ns }}$ & 0,0007 \\
CRMF & 34767,98 & 21,97 & $200988967,79^{* *}$ & 58359022,98 \\
CRF & 4299,48 & 17,71 & $1489399,98^{*}$ & 568908,58 \\
CRG & 836,88 & 16,92 & $50506,00^{*}$ & 20062,26 \\
ARMF & 41,55 & 42,11 & $633,52^{\text {ns }}$ & 306,21 \\
ARF & 222,91 & 19,64 & $4831,64^{*}$ & 1916,73 \\
ARG & 232,07 & 16,34 & $3987,08^{*}$ & 1438,37 \\
MSPA & 41,55 & 42,22 & $688,56^{\text {ns }}$ & 307,76 \\
MSR & 5,4511 & 29,31 & $3,73^{\text {ns }}$ & 2,55 \\
MST & 0,8811 & 21,57 & $0,0703^{\text {ns }}$ & 0,0361 \\
MSR/MSPA & 6,3322 & 27,59 & $4,5521^{\text {ns }}$ & 3,0524 \\
CE & 0,1743 & 20,03 & $0,0020^{\text {ns }}$ & 0,0012 \\
FR & 6084,61 & 21,19 & $2384749,38^{\text {ns }}$ & 1663859,13 \\
DeTR & 1007,58 & 14,59 & $35714,07^{\text {ns }}$ & 21625,87 \\
\hline & 0,1689 & 12,02 & $0,0005^{\text {ns }}$ & 0,0004 \\
\hline
\end{tabular}

$* *, *=$ Significativo ao nível de 1 e $5 \%$, respectivamente, pelo teste $\mathrm{F}$;

ns $=$ Não significativo

${ }^{1}$ Comprimento total de raiz $(\mathrm{CTR})(\mathrm{cm})$; Área de superfície total de raiz $(\mathrm{ATR})\left(\mathrm{cm}^{2}\right)$; Diâmetro médio de raiz (DMR) (mm); Volume total de raiz (VTR) $\left(\mathrm{cm}^{3}\right)$; Número de ramificações (NR); Comprimento de raiz muito fina $(\mathrm{CRMF})(\mathrm{cm})$; Comprimento de raiz fina $(\mathrm{CRF})(\mathrm{cm})$; Comprimento de raiz grossa $(\mathrm{CRG})(\mathrm{cm})$; Área de superfície de raiz muito fina (ARMF) $\left(\mathrm{cm}^{2}\right)$; Área de superfície de raiz fina (ARF) $\left(\mathrm{cm}^{2}\right)$; Área de superfície de raiz grossa $(\mathrm{ARG})\left(\mathrm{cm}^{2}\right)$; Massa seca de parte aérea (MSPA) (g 3plantas $\left.{ }^{-1}\right)$; Massa seca de raiz (MSR) (g 3plantas $\left.{ }^{-1}\right)$; Massa seca total (MST) (g 3plantas $\left.{ }^{-1}\right)$; relação raiz/parte aérea (MSR/MSPA); Comprimento específico (CE) (cm g-1); Finura de raiz (FR) $\left(\mathrm{cm} \mathrm{cm}^{-3}\right)$; Densidade de tecido de raiz (DeTR) $\left(\mathrm{g} \mathrm{cm}^{-3}\right)$.

5), fazendo com que os valores de CE para essas linhagens sejam superiores as demais, o que pode estar relacionado com uma diferença no padrão de alocação de fotoassimilados nas raízes dessas plantas, podendo estar formando aerênquimas em seus tecidos radiculares. Entre as linhagens eficientes, somente a SC283 apresentou média inferior à linhagem ineficiente BR007B para CE. Esses resultados indicam uma possível relação entre eficiência 
e CE em sorgo, sinalizando para a importância de aspectos rizoeconômicos (custo de massa radicular por potencial retorno) na adaptação das plantas de sorgo em ambientes de estresse de fósforo (Ryser, 2006).

A característica que apresentou uma tendência em separar as linhagens eficientes das ineficientes foi a densidade de tecido de raiz (DeTR) (Tabela 5). As linhagens eficientes apresentaram uma tendência a valores médios menores que as linhagens ineficientes (Tabela 5), indicando que as plantas com superior adaptação tendem a desenvolver raízes com uma menor densidade dos seus tecidos, reduzindo, assim, o gasto metabólico com a taxa respiratória das raízes. Essa característica está relacionada com características anatômicas das raízes, como a formação de aerênquima, e com a taxa de crescimento radicular (Lynch \& Ho, 2005) e pode estar relacionada com a eficiência das linhagens de sorgo cultivadas em baixa disponibilidade de fósforo.

A eficiência de aquisição foi duas a três vezes mais importante que a eficiência de utilização em plantas de milho cultivadas em baixo nível de fósforo, para a eficiência de uso (Parentoni, 2008). A combinação de aquisição e translocação de Pi eficientes é essencial para as plantas manterem adequados os níveis de Pi celular, para o normal funcionamento (Raghothama \& Karthikeyan, 2005). Isso demonstra e reitera a importância da presença de características morfológicas do sistema radicular que favorecem a absorção de nutrientes, refletindo no desenvolvimento das plantas cultivadas em ambientes com baixa disponibilidade de fósforo.

\section{Agradecimento}

Os autores agradecem à Embrapa, à Fundação McKnight e à Fundação de Amparo à Pesquisa do Estado de Minas Gerais - Fapemig o apoio financeiro.

\section{Literatura Citada}

BHOM, W. Methods of studying root systems. New York: Springer -Verlag, 1979. 189 p.

BOUMA, T. J.; NIELSON, K. L.; KOUTSTAAL, B. A. S. Sample preparation and scanning protocol for computerized analysis of root length and diameter. Plant and Soil, The Hagues, v. 218, p. 185-196, 2000.

CASPER, B. B.; JACKSON, R. B. Plant competition underground, Annual Review Ecology and Systematic, Palo Alto, v. 28, p. 545-570, 1997.

CLARKSON, D. T.; HAWKESFORD, $M$. J. Molecular biological approaches to plant nutrition. Plant and Soil, The Hague, v. 155/156, p. 21-31, 1993.

DESNOS, T. Root branching responses to phosphate and nitrate. Plant Biology, Stuttgart, v. 11, p. 82-87, 2008.

RODRIGUES, J. A. S. (Ed.). Cultivo do Sorgo 3. ed. Sete Lagoas: Embrapa Milho e Sorgo, 2007. (Embrapa Milho e Sorgo. 
Sistemas de Produção, 2). Disponivel em: < http://sistemasdeproducao.cnptia.embrapa. br/FontesHTML/Sorgo/CultivodoSorgo_3ed/ index.htm> Acesso em: 01 jul 2007

FAGERIA, N. K. Otimização da eficiência nutricional das culturas. Revista Brasileira de Engenharia Agrícola Ambiental, Campina Grande, v. 2, p. 6-16, 1998.

FITTER, A. H. The ecological significance of root system architecture: an economic approach. In: ATKINSON, D. (Ed.) Plant Root Growth: an ecological perspective. London: Blackwell Scientific, 1991. p. 229-243.

GAHOONIA, T. S.; NIELSEN, N. E. Root traits as tools for creating phosphorus. efficient crop varieties. Plant and Soil, The Hagues, v. 260, p. 47-57, 2004.

GERKE, J.; RÖMER, W.; JUNGK, A. The excretion of citric and malic acid by proteoid roots of Lupinus albus L.; effects on soil solution concentrations of phosphate, iron, and aluminumin the proteoid rhizosphere in samples of an oxisol and a luvisol. Journal of Plant Nutrition and Soil Science, Weinheim v. 157, p.289-294, 1994.

GOOD, A. G.; SHRAWAT, A. K.; MUENCH, D. G. Can less yield more? Is reducing nutrient input into the environment compatible with maintaining crop production? Trends in Plant Science, Oxford, v. 9, p. 597-605, 2004.

JAIN, A.; POLING, M. D.; KARTHIKEYAN, A. S.; BLAKESLEE, J. J.; PEER, W. A.; TITAPIWATANAKUN, B.; MURPHY, A. S.; RAGHOTHAMA, K. G. Differential effects of sucrose and auxin on localized phosphate deficiency-induced modulation of different traits of root system architecture in Arabidopsis. Plant Physiology, Bethesda, v. 144, p. 232-247, 2007b.

KHAMIS, S.; CHAILLOU, S.; LAMAZE, T. $\mathrm{CO}_{2}$ assimilation and partitioning of carbon in maize plants deprived of orthophosphate. Journal of Experimental Botany, London, v. 41, p. 1619-1625, 1990.

LAMBERS, H.; SHANE, M.W.; CRAMER, M. D.; PEARSE, S. J.; VENEKLAAS, E.J. Root structure and functioning for efficient acquisition of phosphorus: matching morphological and physiological traits. Annals of Botany, London, v. 98, p. 693-713, 2006.

LIU, G.; DUNLOP, J.; PHUNG, T.; LI, Y. Comparisons of two quick methods for evaluating phosphorus efficiency genotypes. In: INTERNATIONAL SYMPOSIUM ON PHOSPHORUS DYNAMICS IN THE SOILPLANT CONTINUUM, 3., 2006. Uberlândia. Proceedings... Sete Lagoas: Embrapa Milho e Sorgo, 2006. p. 100-101.

LYNCH, J. P.; HO, M. D. Rhizoeconomics: carbon costs of phosphorus acquisition. Plant and Soil, The Hague, v. 269, n. 1-2, p. 45-56, 2005.

MARSCHNER, H. Mineral nutrition in plants. 2nd ed. San Diego: Academic Press, , 1995. $889 \mathrm{p}$.

NOVAIS, R. F.; MELLO, J. W. V. Relação Solo-Planta. In: NOVAIS, R. F.; ALVAREZ 
V., V. H.; BARROS, N. F. de; FONTES,R. L. F.; CANTARUTTI, R. B.; NEVES, J. C. L. (Ed.). Fertilidade do solo. Viçosa, MG: Sociedada Brasileira de Ciência do Solo, 2007. p. 133-204.

PARENTONI, S. N. Estimativas de efeitos gênicos de diversos caracteres relacionados à eficiência e resposta ao fósforo em milho tropical. 2008. 207 f. Tese (Doutorado em Agronomia - Genética e Melhoramento de Plantas) - Escola Superior de Agricultura Luiz de Queiroz, Universidade de São Paulo, Piracicaba

RAGHOTHAMA, K. G.; KARTHIKEYAN, A. S. Phosphate acquisition. Plant and Soil, The Hague, v. 274, p. 37-49, 2005.

RENGEL, Z.; MARSCHNER, P. Nutrient availability and management in the rhizosphere: exploiting genotypic differences. New Phytologist, Oxford, v. 168, p. 305-312, 2005.

REYMOND, M.; SVISTOONOFF, S.; LOUDET, O.; NUSSAUME, L.; DESNOS, T. Identification of QTL controlling root growth response to phosphate starvation in Arabidopsis thaliana. Plant Cell and Environment, Oxford, v. 29, p.115-125, 2006.

RYSER, P. The mysterious root length. Plant and Soil, The Hague, v. 286, n 1-2, p. 1-6, 2006.

SANCHEZ-CALDERON, L.; LOPEZ-BUCIO, J.; CHACON-LOPEZ, A.; CRUZ-RAMIREZ, A.; NIETO-JACOBO, F.; DUBROVSKY, J. G.; HERRERA-ESTRELLA, L. Phosphate starvation induces a determinate developmental program in the roots of Arabidopsis thaliana. Plant and Cell Physiology, Tokyo, v. 46, p.174184, 2005.

SCHAFFERT, R. E.; ALVES, V. M. C.; PITTA, G. V. E.; BAHIA FILHO, A. F. C.; SANTOS, F. G. Genetic variability in sorghum for P efficiency and responsiveness, In: INTERNATIONAL PLANT NUTRITION COLlOQUIUM, 2001, Netherlands. Plant nutrition: food security and sustainability of agro-ecosystems - proceedings. Dordrecht: Kluwer, 2001. p. 72-73,.

SCHROEDER, M. S.; JANOS, D. P. Plant growth, phosphorus nutrition, and root morphological responses to arbuscular mycorrhizas, phosphorus fertilization, and intraspecific density. Mycorrhiza, Berlin, v. 15, p. 203-216, 2005.

SHARPLEY, A. N.; MCDOWELL, R. W.; KLEIMMAN, P. J. A. Phosphorus loss from land to water: integrating agriculture and environmental management. Plant and Soil, The Hague, v. 237, p. 287-307, 2001.

WILCOX, C. S.; FERGUSON, J. W.; FERNANDEZ, G. C. J.; NOWAK, R. S. Fine root growth dynamics of four Mojave Desert shrubs as related to soil moisture and microsite. Journal of Arid Environments, London, v. 56, p. 129-148, 2004.

WILLIAMSON, L. C.; RIBRIOUX, S. P.; FITTER, A. H.; LEYSER, H. M. Phosphate availability regulates root system architecture in Arabidopsis. Plant Physiology, Bethesda, v. 126, p. 875-882, 2001. 